\title{
Erratum: Dephasing of Majorana-based qubits [Phys. Rev. B 97, 125404 (2018)]
}

Christina Knapp, Torsten Karzig, Roman M. Lutchyn, and Chetan Nayak

Q (Received 6 July 2018; published 17 July 2018)

DOI: 10.1103/PhysRevB.98.039903

Equation (41) in the original article has an extra factor of $L / \xi$ and should read

$$
T_{2, \beta}^{*}=\tau_{0} \exp \{\beta \Delta\} .
$$

This changes our estimates in the third row of Table I to $T_{2, \beta}^{*}=20 \mathrm{~s}$ for all values of $L / \xi$. Additionally, Eq. (E3) in the original article has the same error and should read

$$
T_{2, \beta}^{*}{ }^{-1}=\sqrt{\frac{\pi}{2 \Delta \beta}} S_{\mathrm{ph}}(-\Delta) .
$$

These changes do not affect the main results, discussion, or conclusion of the article. 